\title{
LA SEXUALITÉ À MI-CHEMIN ENTRE L'INTIMITÉ ET LE GRAND PUBLIC
}

\section{Lubomira Radoilska}

Presses Universitaires de France | « Cités »

2003/3 $n^{\circ} 15 \mid$ pages 31 à 42

ISSN 1299-5495

ISBN 9782130534563

Article disponible en ligne à l'adresse :

https://www.cairn.info/revue-cites-2003-3-page-31.htm

Distribution électronique Cairn.info pour Presses Universitaires de France.

(C) Presses Universitaires de France. Tous droits réservés pour tous pays.

La reproduction ou représentation de cet article, notamment par photocopie, n'est autorisée que dans les limites des conditions générales d'utilisation du site ou, le cas échéant, des conditions générales de la licence souscrite par votre établissement. Toute autre reproduction ou représentation, en tout ou partie, sous quelque forme et de quelque manière que ce soit, est interdite sauf accord préalable et écrit de l'éditeur, en dehors des cas prévus par la législation en vigueur en France. Il est précisé que son stockage dans une base de données est également interdit. 


\title{
La sexualité à mi-chemin entre l'intimité et le grand public
}

\author{
LUBOMIRA RADOILSKA
}

\begin{abstract}
"Espace privé et espace public s'imbriquent. Vie privée et vie publique se percutent. Intimité et "extimité" se combinent. For intérieur et for extérieur se répondent. Toutefois, tandis que l'espace privé, la vie privée et maintenant l'intimité accèdent à la visibilité, le for intérieur demeure, pour l'instant, à l'abri des feux de la rampe. Les cas de conscience, l'introspection, la réflexion sur les mobiles, l'irrésolution se nouent hors du regard public, demeurent (encore ?) à l'ombre. Les témoins de la télévision de l'intimité l'attestent : tout en s'exposant, ils redélimitent leur "jardin caché". Tout en se livrant, ils préservent leur singularité. Ils offrent en spectacle, et pour les besoins du spectacle, des fragments de leur personnalité qui, selon eux, ne leur appartiennent pas en propre. Leur quant-à-soi sort intact de ses épreuves. »
\end{abstract}

Dominique Mehl, La télévision de l'intimité, Paris, Éd. du Seuil, 1996, p. 234-235.
31

La sexualité à mi-chemin entre lintimité et le grand public L. Radoilska

L'apparition au début des années 1990 des reality shows à la télévision française a fait objet des recherches sociologiques minutieuses, qui ont conclu à l'impossibilité de distinguer entre vie privée et vie publique, ces deux concepts étant soumis à une redéfinition permanente, elle-même socialement changeante. Ce qui appartient au domaine de l'intime, au noyau dur de la vie privée, n'apparaît plus comme un lieu commun, et n'est identifiable que dans le cadre d'une démarche fortement individualisée. Pourtant, cette démarche s'effectue souvent sous l'emprise d'un "besoin de se dire et de se montrer sous son identité la plus ordinaire " (D. Mehl, op. cit., p. 10) et, par ce fait même, se constitue en sollicitation impérative d'une réponse sociale, voire d'un aval public.

Cités 15, Paris, PUF, 2003 
Comment rendre compte de ces mouvements de fluctuation entre la vie privée et la vie publique ? La question semble encore plus difficile à aborder, dix ans plus tard, compte tenu des phénomènes qui, comme "Loft Story ", mettent en scène des vies privées élues (selon quels critères d'ailleurs ?), destinées à se dérouler sous l'œil intéressé du grand public.

S'agit-il d'une transformation irrévocable de la manière dont on considère l'intimité ? Avons-nous de bonnes raisons pour croire que l'intimité d'aujourd'hui est plus transparente qu'elle ne l'était auparavant, plus exposable et ouverte, moins secrète ? L'hypothèse que je vais explorer ici est que, malgré les apparences, le sens de l'intime n'a pas considérablement changé, alors que, en revanche, l'extension de ce terme a subi des rétrécissements et des remodelages importants. Tandis que l'intimité reste toujours un espace restreint, peut-être même plus qu'avant, ses références sont devenues de plus en plus aléatoires et instables. En particulier, on peut se demander si la sexualité fait toujours et nécessairement partie de la vie intime, au vu d'une certaine facilité à mettre en évidence son propre vécu sexuel et à en discuter ouvertement, une facilité inconnue auparavant et incontestablement omniprésente aujourd'hui. Je tiens à préciser que les réflexions qui suivront n'ont aucune prétention sociologique et se limitent à une analyse conceptuelle et philosophique basée exclusivement sur deux œuvres - l'une cinématographique, l'autre littéraire - prises en tant que témoignages.

Dossier:

Par rapport aux représentations récentes de l'intimité au cinéma, le documentaire de Daniel Karlin Et si on parlait d'amour... constitue un cas particulier et aussi extrêmement intéressant dans la mesure où ce film ne relève pas de la fiction, mais, précisément, du témoignage. Le spectateur est invité à découvrir, jusqu'au point d'y assister, la vie privée des " acteurs" qui sont des personnes tout à fait réelles et identifiables. Cet élément me paraît très important, parce qu'il efface en grande partie la distance entre le réel et l'image, et ce d'une manière plus radicale et efficace qu'une représentation fictionnelle, si militante qu'elle soit, comme par ex., Baise-moi ou Romance. Dans ce sens, il me semble légitime de dire que le message du documentaire de D. Karlin est beaucoup plus subversif que celui de ces films extrêmement débattus.

De fait, ce que l'on voit dans Et si on parlait d'amour... est censé bouleverser l'image que nous nous faisons de la normalité dans la sphère intime. D'une part, des gens ordinaires assument sans difficulté des activités sexuelles, considérées d'habitude comme marginales, pour ne pas dire 
perverses. Ils ne se cachent pas et sont convaincus de ne rien avoir à cacher. D'autre part, des personnes handicapées, exclues de la communication amoureuse d'après l'opinion commune, montrent les mêmes attentes et les mêmes attitudes affectives et sensuelles que le reste du monde.

Mais quelle finalité possède la représentation frappante de ce contraste? Dans un entretien publié dans Le Nouvel Observateur ( $\mathrm{n}^{\circ} 1953,2002$, p. 102-104), D. Karlin affirme avoir fait un film moral contre l'hypocrisie et au nom de la transparence. Ainsi, le documentaire Et si on parlait d'amour... s'inscrirait dans une logique dont l'idée régulatrice n'est autre chose qu'un monde social où, selon les mots du réalisateur, "les gens disent ce qu'ils font et font ce qu'ils disent dans le cadre du respect d'autrui et du respect de la loi ».

Que devient la vie intime si l'on suit cette logique ? Pour répondre à cette question, il faudrait savoir quel type d'opposition lie les deux termes majeurs de la réflexion de Karlin, à savoir l'hypocrisie et la transparence. Sont-ils contradictoires ou seulement contraires? Autrement dit, y a-t-il des options intermédiaires ou doit-on adopter soit l'hypocrisie, soit la transparence? L'enjeu est extrêmement important, puisque, dans le cas du tiers exclu, on se voit imposer, sur le plan de l'action, un devoir de transparence aussi intransigeant que le devoir de vérité sur le plan de la logique. Cette analyse donne lieu à un dilemme intéressant.

L'interprétation forte de la relation entre l'hypocrisie et la transparence peut justifier le propos de Karlin. Elle explique l'intérêt qu'il peut y avoir à montrer sur le grand écran, par exemple, une longue scène de fellation chez un couple de handicapés ou une partouze qui voit une épouse cinquantenaire faire l'amour avec trois autres personnes, tandis que son mari prend des photos pour les prochaines annonces échangistes, tout en jouant le rôle d'animateur. Si l'on ne se veut pas hypocrite, on doit se situer du côté de la transparence. Mais sommes-nous prêts à assumer toutes les conséquences d'une telle position, qui rend obsolètes des catégories comme intimité, secret, droit au silence, et semble aboutir à un monde social assez orwellien?

En revanche, l'interprétation faible du rapport entre l'hypocrisie et la transparence ne suffit pas pour justifier un film comme Et si on parlait d'amour... qui semble se dérouler dans l'ignorance d'une option fondamentale qui est celle d'être contre l'hypocrisie et contre la transparence. Pourtant, elle serait la seule capable de garantir la possibilité d'une vie 
intime, si l'on souhaite rester dans l'opposition d'hypocrisie et de transparence.

Encore faut-il se poser la question de savoir si cette opposition en est vraiment une. Je ne vois pas en quoi le fait de "tout dire " et de "tout montrer " empêche la déception, y compris de soi-même, si l'on admet que la transparence peut être aussi trompeuse que toute forme classique de l'hypocrisie.

Les « acteurs " de Et si on parlait d'amour... ne semblent pas partager ces doutes, même si le souci de vérité et, surtout, celui de transparence ne leur sont nullement étrangers. Ils prononcent souvent des mots de cet ordre pour expliquer leur mode de vie. Ils ne mentent à personne, ils se disent même incapables d'avoir une histoire derrière le dos de leurs conjoints, ils sont honnêtes. De même, le genre de rapports sexuels qu'ils expérimentent est connu dans leur milieu social et professionnel, sans qu'ils en subissent un quelconque préjudice moral. La conclusion qui semble s'imposer est que "tout va bien ", pourvu que l'on ne cache rien, ne force personne et que l'on sache et fasse ce que l'on veut.

Précisément, le fait que "tout va bien ", représente, à mon avis, un élément majeur de Et si on parlait d'amour... Ce film met en scène des personnes performantes dans leur cadre professionnel, accomplies dans leur vie privée, parfaitement à l'opposé des obsédés, des pervers, des

Dossier: Politiques de la pornogratapie marginaux. Or, si, comme le démontre Dominique Mehl, une des motivations fondamentales des premiers reality shows était de donner la parole aux gens pour crier leur malaise, le documentaire de Karlin étale, par contre, des témoignages de bien-être, qui envoient en l'air (si l'on peut dire) le modèle idéologique et moral de l'amour unique que le cinéaste déplore dans son interview.

Ne serait-il alors pas plus approprié de mesurer l'effet de ce film non pas à un absurde devoir de transparence, mais à un droit de transparence tout à fait légitime? Mais en vertu de quoi pourrait-on le reconnaître ? Car, de ce dernier droit, il semble exclu qu'il soit simplement justifié par la crainte que la société soit intolérante vis-à-vis des personnes qui, comme les héros du film, sont adeptes de l'amour à plusieurs. Cela nous conduit à suivre une autre piste, qui remet le droit de transparence dans le cadre de tout un projet de libération.

La cible du documentaire de Karlin serait alors une image élitiste et démodée de l'amour. Ce modèle décourageant postule que, pour nous faire aimer, nous devons posséder tout un arsenal de qualités dont la 
plupart dépendent assez peu de nous, comme la beauté, par exemple, et, de surcroitt, avoir la chance de rencontrer l'âme sœur, une chance qui tient au miracle. Rien de tout cela dans le film de Karlin. Au contraire, il suffit de vouloir faire l'amour et de se rendre là où il le faut : sur un site internet ou dans une boîte de nuit. Et si on parlait d'amour... traite de la facilité des rencontres sexuelles comme s'il s'agissait d'une réelle démocratisation de l'épanouissement personnel. Le supposé droit de transparence serait alors révélateur de cette aisance bénéfique.

Pourtant, les «acteurs » de Karlin suivent le credo de tout dire et de ne rien cacher d'une manière hautement sélective. Il est vrai que, par rapport à la sexualité, il ne reste plus grand-chose à montrer. On dévoile entièrement les façons dont on dispose de son corps, et on rend publique sa jouissance. Tout de même, il y a des non-dits surprenants dans ce cadre de transparence. Pour donner un exemple, je vais introduire quelques éléments de la première histoire du film, "La famille ".

C'est l'histoire d'un couple, Bernard, ancien instituteur et candidat de la gauche aux dernières municipales, et Violette, aide-soignante, qui pratiquent l'échangisme depuis presque trente ans et ont gardé leur mariage intact. Aux yeux de leurs proches, ils représentent un idéal d'honnêteté, puisqu'ils ne se trompent pas mutuellement et ne se cachent rien. La famille du couple, les petits-enfants compris, est au courant de leurs expériences échangistes : tout le monde en discute librement à table. On peut entendre les petits enfants se prononcer sur la vie sexuelle de leurs grandsparents : il y en a certains qui auraient préféré de ne pas en connaitre les détails, mais tous affirment ne voir rien de mal dans ce que mamie et papi font, puisque c'est leur façon de s'amuser. Le courant passe aussi avec l'arrière-grand-mère à qui l'on offre un "zizi-sauteur " avec le café. Elle affirme approuver sans réserve l'intimité transparente du couple de sa fille, mais, attention, elle ne lui a jamais annoncé le nom de son père biologique. Pour quelle raison la mère de Violette ne dévoile-t-elle pas l'identité du géniteur ? Tout simplement, elle veut garder ce secret pour elle-même. Cela ne concerne personne d'autre, dit-elle. Et, d'ailleurs, Violette ne pose même pas la question. Pourquoi ? Cela n'a pas d'importance pour elle? Violette ne répond pas, elle se met à pleurer.

À la lumière de ce secret de famille gardé au long d'un demi-siècle, le fait de raconter et de montrer sa vie sexuelle avec tant d'aisance prend un tout autre sens que celui d'une chasse à l'hypocrisie par la mise en lumière de sa vie intime. Une telle facilité ne démontrerait que la banalisation de 
l'acte sexuel, et nullement une nouvelle attitude par rapport à l'intimité. La tendance de libéralisation des rapports sexuels que le film de D. Karlin représente a donc une contrepartie difficile à avaler : la sexualité serait libre de tout poids affectif et donc ne ferait plus partie importante de notre vie intime, l'intimité véritable étant ailleurs.

Tout au long de Et si on parlait d'amour..., on trouve des indices de ce changement radical des rapports entre affection et intérêt sexuel. Plus il y a de distance entre ces deux expériences, plus le dévoilement de ses propres pratiques sexuelles devient inoffensif. Pour les traiter et les manifester de cette façon détachée, il faut d'abord les confronter à des activités qui n'ont pas vraiment de dimension personnelle. Si Julien, un des personnages de Karlin et père de trois enfants, nous apprend qu'il a essayé ce que cela donnait de se faire sodomiser et de faire une fellation pour comprendre que, en fin de compte, il préférait les filles, saurions-nous quelque chose de plus important sur lui que s'il nous avait dit qu'il préférait le café expresso et que, afin de bien s'en rendre compte, il avait goûté d'autres types de café ? La banalisation de l'acte sexuel que je perçois dans le film de D. Karlin passe par l'adoption d'un concept de safe sex, dans un sens très spécifique. Là, il n'est plus seulement question de prendre des mesures contraceptives, et de se protéger contre le sida et les MST. L'essentiel consiste à éviter des répercussions affectives. Ainsi, pour ne pas

Dossier: Politiques de la pornographie entrer dans des ambiguïtés d'ordre sentimental, Julien a consciencieusement cherché un couple homosexuel pour faire ladite expérience. Dans une autre perspective, Cathy, l'hérö̈ne de la deuxième histoire du film, nous explique que l'amour et la tendresse ne sont pas la vocation des rencontres d'une nuit à plusieurs qu'elle vit régulièrement depuis quelque temps. D'après Cathy, ces expériences sexuelles ont pour objectif un plaisir brutal, le côté affectif se situant ailleurs - par exemple, dans une amitié d'enfance.

Mais si la vie sexuelle n'est pas nécessairement liée à la vie intime, la pornographie peut devenir juste un des éléments du quotidien. On peut voir Julien choisir une cassette porno pour la soirée en famille sans plus d'état d'âme que ses enfants qui choisissent leur dessin animé. Plus tard, Sophie, son épouse, qui arrive et a raté le début du film, demande à Julien de lui raconter "ce qui c'est passé " tout comme s'il s'agissait du soap de l'après-midi.

Cette distance entre sentiments amoureux et attitudes sexuelles se laisse entendre également dans la façon dont les acteurs de Karlin défendent à 
plusieurs reprises l'idée de la fidélité conjugale. Malgré leurs expériences sexuelles hors couple, Violette et Bernard, Sophie et Julien, tous se disent fidèles à leurs conjoints. Une jolie image de la fidélité affective en toute transparence, mais qui entraîne le fait de ne plus concevoir l'acte d'amour éminemment comme une expression d'amour.

Revenons au supposé effet libérateur du film lié au concept de la démocratisation du plaisir sexuel et, par là, à un certain concept sous-jacent de l'épanouissement personnel. Il me semble pertinent de dire que Et si on parlait d'amour... pourrait s'inscrire dans une logique de libération d'un des préjugés contemporains concernant le corps humain : mais au prix de retomber sous l'emprise d'un autre préjugé. Pour éclaircir ce point, je me réfere à l'ouvrage de Michela Marzano récemment paru, Penser le corps (PUF, 2002). Dans les termes de cet ouvrage et de la réflexion cohérente qu'il propose sur le corps, le film de Karlin serait libérateur par rapport à la hantise du "corps idéal ", tout en réaffirmant la validité du "corpsinstrument».

Qu'est-ce que définit le concept du corps idéal ? En commençant par la publicité et les corps jeunes et parfaits des mannequins, ce concept vise à expliquer des phénomènes sociaux comme le développement de la chirurgie esthétique et la fixation sur l'apparence du corps, qui finit par produire des états morbides du type de l'anorexie et de la boulimie. Mais quel est le rapport de ce concept avec Et si on parlait d'amour... ? En fait, l'opposition fervente à l'obsession du corps idéal me paraît être la réelle inspiration de ce film. Les personnes qui exposent leur nudité devant la caméra de Karlin sont extrêmement loin du physique parfait. Et c'est peut-être cet élément qui fait, à lui seul, la force de leur témoignage. On peut être désiré et aussi véritablement aimé sans avoir le corps sans âge et mince des mannequins, ou le corps musclé et toujours sans âge des athlètes. On peut même être promis à une chaise roulante pour le reste de ses jours sans pour autant que cela implique que l'on n'aura plus de vie amoureuse. Comme le dit avec justesse Karine, une des actrices de Karlin, qui, à cause de sa maladie, n'a jamais pu marcher sur ses pieds, on a tous été éduqués à vouloir être aimés, et il n'y a pas de raison pour y renoncer, même lorsqu'un handicap est présent dans sa vie.

Mais la question qui se pose est de savoir s'il faut complètement s'abstraire du corps comme valeur esthétique et éthique pour aboutir à cette liberté d'esprit. Je crains en effet que cela ne fasse partie du message de Karlin. Tout au moins, c'est une conséquence logique du fait que l'on 
ne s'identifie plus à son corps, puisque l'on en dispose dans l'optique sexuelle sans limite aucune, pourvu que les autres participants soient consentants.

De ce fait, il me semble qu'il existe une confusion conceptuelle implicite dans Et si on parlait d'amour... On commence par le constat d'une libéralisation des usages sexuels du corps traité comme l'instrument par excellence du plaisir. On continue en se félicitant du fait que les rapports sexuels avec des partenaires inconnus sont tout à fait satisfaisants et impliquent le sentiment d'être recherché, accepté, voire aimé. On passe par l'identification de ce sentiment non seulement avec le sentiment de bienêtre, mais aussi avec la réalité même du bien-être. Et on finit par se convaincre que, puisque l'on se sent bien et qu'on ne contrarie personne, on a de bonnes raisons pour mener une telle vie, et aucune pour s'y abstenir.

Quels sont les éléments problématiques de cette ligne de réflexion ? Il me paraît qu'elle consiste à établir un passage illicite de la description à la justification des phénomènes présentés. Par cela, je ne veux pas dire que tout passage de ce genre soit illicite. En conséquence, je me contenterai d'expliciter les raisons qui me font croire en la présence de quelques courts-circuits inquiétants dans le film de Daniel Karlin.

En particulier, je suis restée très perplexe du statut accordé au corps Dossier: Politiques de la pornographie humain. Je ne vois pas comment la facilité et le pluralisme des rapports sexuels que ce film montre seraient possibles sans une sorte de conviction que l'être humain possède tout simplement son corps, sans s'y identifier. Mais, si le corps n'est rien d'autre qu'un instrument docile du plaisir, il n'y a aucun lien essentiel entre les expériences sexuelles, aussi réussies et intéressants soient-elles, et la vie affective ou amoureuse. Tout au plus, elles peuvent de temps en temps coïncider. Dans ce cas, certaines facettes du vécu sexuel feraient encore partie de la vie intime, tandis que d'autres (la majorité, peut-être) en seraient complètement coupées. En ce sens, on peut facilement accorder le fait qu'il n'y a rien d'exclusif dans les rapports sexuels, principe sur lequel Et si on parlait d'amour... semble insister. Mais, puisque ces rapports ne sont plus l'expression authentique des sentiments amoureux, cela ne signifie pas que l'amour est devenu une relation moins exclusive qu'avant. Ce film ne démontre donc pas l'apparition d'un "nouvel ordre amoureux » comme l'annonce le titre même de l'interview de Karlin pour Le Nouvel Observateur, encore moins l'avènement d'une intimité plus transparente. 
En revanche, le film joue sur un quiproquo entre amour et faire l'amour, entre la recherche d'être aimé et la recherche de nouvelles expériences sexuelles. Cela permet d'exagérer l'importance de ces expériences qui, tout au contraire, démontrent la marginalisation de la sexualité par rapport à la vie intime.

Mais où, alors, s'effectue le glissement du témoignage à la justification ? Ce malentendu vient, à mon avis, non seulement de la confusion de l'expérience amoureuse et de l'expérience sexuelle, préalablement dissociées, mais aussi, et je crois surtout, de l'identification infondée du plaisir et de la satisfaction à l'épanouissement personnel et au bien-être. Effectivement, une partie importante du film de Karlin est consacrée à démontrer que ses acteurs sont des gens équilibrés, épanouis et que leurs pratiques sexuelles font partie de leur accomplissement. L'argument consiste à dire que, si une chose rend heureux quelqu'un et si cette chose n'implique pas nuire aux autres, alors, elle est bonne ou, au moins, incritiquable.

Cependant, si l'on accepte cet argument, on s'oblige également à prendre pour un critère de la vie accomplie le sentiment de satisfaction ou même de bien-être, un sentiment subjectif qui ne tient pas compte de l' "élément propositionnel " du bonheur qui s'exprime par la formule "être heureux du fait que ». Où est le problème ? On doit quand même reconnaître la capacité de chacun de juger s'il est heureux ou pas. Dans un certain sens, évidemment. Mais, dans un autre qui est d'ailleurs le seul réellement moral, non. Parce que la condition de la satisfaction ressentie est facile à remplir, sans pour autant conduire au bonheur. Pour illustrer ce point, je me permets d'emprunter un exemple (extrême) de l'article de Philippa Foot "La vertu et le bonheur » : la vie d'une personne lobotomisée qui passe toutes ses journées, parfaitement heureuse, à ramasser des feuilles dans le jardin de l'hôpital. Ainsi, le sentiment d'être bien dans leur peau qu'éprouvent les acteurs de Karlin ne suffit pas pour établir si leurs vies sont réellement accomplies.

Dans cette optique, on peut se demander si une femme qui ignore l'identité de son père, à qui son mari a toujours refusé le droit d'enfanter par peur qu'elle ne préfere pas leurs enfants aux enfants qu'il a eus de son précédent mariage, et qui pratique l'échangisme depuis trente ans pour éviter que son mari aille voir ailleurs, mène réellement une vie heureuse. On peut se demander si, au lieu de défier l'hypocrisie, la transparence telle que l'entend Et si on parlait d'amour... ne fait plutôt reculer l'intimité réelle, et le rapport à soi. Un résultat paradoxal qui tient à l'identification 
illusoire de "tout dire" et de "ne rien cacher". Plus de visibilité ne signifie donc pas nécessairement plus de compréhension.

En analysant Et si on parlait d'amour..., j'ai essayé de montrer des indices que l'expérience sexuelle en tant que telle n'a plus de connotation intime stable. Mais elle n'est pas séparée de la vie intime une fois pour toutes, non plus. Il s'agit en fait d'une ambiguïté fondamentale déjà implicite au terme d'expérience et multipliée par l'incertitude exacerbée quant au statut du corps humain. Bien entendu, une expérience sexuelle peut représenter du vécu personnel et avoir une extrême importance pour la constitution de soi par rapport à l'autre. Cependant, il est également possible que l'on conçoive ses expériences sexuelles comme une sorte d'expérimentation quasi scientifique et, dans ce cas, pourquoi les garder pour soi-même? Dans la perspective de l'expérimentation, la vie sexuelle peut être rendue publique sans que l'on ait le sentiment de s'exhiber ou de faire entrer n'importe qui dans son jardin secret. Or ce n'est pas par le fait que l'on en parle ouvertement ou qu'on la montre sans réserve aucune que la sexualité sort du cadre de l'intime ; elle doit en être déjà émancipée afin que sa publicisation soit devenue possible.

Le film de Karlin oblige donc à faire face à deux compréhensions de la vie sexuelle nettement distinctes et plutôt incompatibles qui, par ailleurs, semblent faciles à confondre. Cette conclusion se précise et confirme par la présentation des rapports entre sexualité, corps et intimité dans le bestseller de Catherine Millet.

Sans doute, par rapport à la tendance de traiter la sexualité comme un champ d'expérimentation, le récit $L a$ vie sexuelle de Catherine $M$. constitue-t-il un témoignage tout à fait précieux. À travers celui-ci, on peut suivre jusqu'au bout la logique de la dissociation entre soi-même et le corps que l'on habite, et estimer au juste ses effets. Si, dans le documentaire de Karlin, il était encore question sinon d'amour, tout au moins de désir et de plaisir comme moteurs des expériences sexuelles, C. Millet nie fermement cette dépendance. L'auteur décrit le fait d'avoir des relations sexuelles et le fait d'éprouver du désir comme étant " presque deux activités séparées » (p. 120) et insiste que, jusqu'à l'âge de 35 ans, c'est-à-dire, pendant les premières dix-sept années de son parcours sexuel extrêmement riche, elle n'a jamais envisagé que son propre plaisir puisse être la finalité d'un rapport sexuel (p. 198).

Les termes que C. Millet choisit pour rendre compte des raisons qui l'ont amenée à participer régulièrement à des pratiques sexuelles assez 
inhabituelles appartiennent à tout un autre registre : "curiosité intellectuelle ", "indifférence aux usages que l'on fait des corps » (p. 73, 203). Il s'agit encore une fois d'une totale "disponibilité du corps et de l'esprit " indispensable pour surpasser les limites imposées par les circonstances, le nombre des partenaires, leurs qualités physiques et morales, même par son propre dégoût et arriver à ce que l'auteur appelle "baiser par-delà toute répugnance " (p. 151).

Le terme de "disponibilité " va de pair ici avec celui d' "endurance ". On y va toujours plus loin, on enlève obstacles après obstacles sans rencontrer de réelle barrière sexuelle, parfois au prix de certaines " misères physiques " à pâtir. L'expérience sexuelle n'est donc pas plaisante, tout au moins, au premier degré. La satisfaction que l'on peut en tirer n'est pas immédiate. Elle s'exprime plutôt dans la conscience d'avoir été capable d'une sublime performance, ou d'avoir obtenu un score bien au-dessus de la moyenne. Faire l'amour s'avère être, avant tout, une discipline physique, un exercice d'endurance continue. En ce contexte, on peut comprendre pourquoi les témoignages de l'extrême contentement des partenaires, pour la plupart d'ailleurs anonymes, jouent un rôle si important dans le récit de C. Millet. Ces témoignages ont incontestablement le statut de preuve, mais aussi celui de récompense, du fait qu'une maîtrise de son propre corps a été effectivement atteinte. Or les activités sexuelles s'inscrivent dans une recherche de "reconnaissance de toute la personne " initialisée au fait par des difficultés de communication (p. 20, 43, 58, 198).

Mais comment peut-on se faire une image cohérente d'une personne qui demande de la reconnaissance en se félicitant du fait qu'elle se laisse " palper et retourner comme une marchandise de choix » (p. 96) ? Cette question parait difficile: ne serait-ce que parce que "cultiver sa propre disponibilité ", le refrain de La vie sexuelle de Catherine $M$., entre en conflit avec la reconnaissance juridique de la personne humaine qui consiste à proclamer justement son indisponibilité. Peut-être ne s'agit-il pas d'une réelle contradiction. Le corps maîtrisé ou plutôt maniable serait la contrepartie de la "liberté fantastique» que C. Millet invoque à plusieurs reprises. Ressentie au sein de l'expérience de se servir de son corps comme d'un instrument parmi d'autres, cette liberté quasiment absolue serait donc la reconnaissance d'un « moi désincarné ». En ce sens, (mal)traiter le corps d'une manière quelconque ne constituerait pas vraiment une atteinte contre la personne humaine qui se définit comme la transcendance propre au corps. 
Cette lecture rend justice à la "dissociation de l'être " dans l'acte sexuel où, pour reprendre les termes de Millet, la conscience soit s'annihile, soit s'échappe laissant le corps à ses automatismes (p. 162). Mais si l'on est de toute façon si peu présent en sa vie sexuelle, la communiquer au grand public ne signifie nullement divulguer ses secrets ou dévoiler son intimité. Justement, vers la fin de son récit, C. Millet remarque qu' " écrire un livre à la première personne relègue celle-ci au rang de la troisième. Plus je détaille mon corps et mes actes, plus je me détache de moi-même. Qui se reconnait dans ces miroirs grossissants qui montrent les joues et le nez comme de vastes terres crevassées ? " (p. 186).

Mais on peut se demander s'il est vraiment possible de vivre à une telle distance radicale de soi-même, c'est-à-dire de son corps. Peut-on pleinement s'identifier à ce moi désincarné afin de jouir effectivement de la liberté illimitée qu'il semble détenir ? D'ailleurs, la nature même de cette liberté relève d'une contradiction au sens strict. Ce qu'elle a d'illimité tient encore une fois à l'effacement de l'opposition entre fantasme et réalité par rapport à l'expérience sexuelle (p. 35). Cependant, il s'agit d'une liberté conditionnée, tributaire des interdits qu'elle brave pour s'y soumettre sous une forme substituée. On a beau s'affranchir de toute interdiction pesant sur les usages du corps la jugeant obsolète, elle revient de force, ses applications changées. L'intimité du corps se dissout dans un environnement bien précis (la chambre commune, le lit " conjugal») et se Dossier: Politiques de la pornographie sédimente autour de certains éléments forts (tout objet intime ou ayant servi un but intime). Une serviette de toilette, celle-ci étant " une sorte d'extension du corps, une prothèse sensible » (p. 159), s'avère plus personnelle que le corps lui-même.

Les résultats de cette analyse confirment, il me semble, mon hypothèse de départ, selon laquelle la facilité de communiquer au grand public son vécu sexuel ne représente pas une nouvelle attitude à l'égard de l'intimité, mais détache la pertinence de ce vécu de toute vie intime. Dans cette perspective, je me demande si l'on ne pouvait pas résumer les témoignages intimistes des deux œuvres présentées ici en une seule affirmation : "Ceci n'est pas mon intimité. " 